\title{
Dark Experiments: From Black Holes to Cosmic Rays
}

\author{
Allen D. Allen \\ Physics Section, New Terra Enterprises, Glorieta, USA \\ Email: allend.allen@yahoo.com
}

Received July 3, 2012; revised August 16, 2012; accepted August 23, 2012

\begin{abstract}
Some nagging questions in modern physics can be resolved rigorously using a basic mathematical formalism, albeit with the need to admit that non-isomorphic realities arise to various degrees in a given universe. Let $U=\left(m^{\prime}, m^{\prime \prime}\right)$ be an unordered pair of distinct massive objects in different reference frames. A dark experiment is an ordering $\left\langle m^{\mu}, m^{v}\right\rangle$ of the elements of $U$, either $\left\langle m^{\prime}, m^{\prime \prime}\right\rangle$ or, exclusively, $\left\langle m^{\prime \prime}, m^{\prime}\right\rangle$, where the left-hand member of the ordered pair is called the observer, and where there exists a 1-to-1 mapping $f:\left\{\left\langle m^{\mu}, m^{v}\right\rangle\right\} \rightarrow\{$ events $\}$, such that both elements of an ordered pair in a dark experiment agree on the events that unfold in the experiment. However, since $\left\langle m^{\prime}, m^{\prime \prime}\right\rangle \neq\left\langle m^{\prime \prime}, m^{\prime}\right\rangle$, it follows that $f\left(\left\langle m^{\prime}, m^{\prime \prime}\right\rangle\right) \neq f\left(\left\langle m^{\prime \prime}, m^{\prime}\right\rangle\right)$. This describes non-isomorphic realities wherein both elements of each ordered pair mapping two distinct sets of unfolding events will agree on their respective events. Consequently, there is an inherent limitation on what can be determined directly from experimentation. Examples arise in the context of the Hawking information paradox, relativistic time travel, and cosmic ray experiments.
\end{abstract}

Keywords: Experimental Physics; Physical Reality; Hawking Information Paradox; Cosmic Rays; Relativistic Time Travel; Lorentz Contraction; Cosmology

Although its roots extend far back in history to philosophical, psychological, and relativistic debates, the concept of what is here referred to as a dark experiment was brought into focus by a recent thought experiment involving a weatherman who jumps out of a spacecraft that is accelerating in the vicinity of a black hole [1]. For the weatherman, this change to a free falling frame negates the existence of the hot stretched horizon due to Hawking radiation. But the hot stretched horizon continues to exist in the accelerating frame, a key to Susskind's resolution of the Hawking information paradox [2]. This leads to the question of what the weatherman reports about the local temperature, and what he himself experiences, in each frame. Indeed, the weatherman will be vaporized when he encounters the stretched horizon in the accelerated frame of the spacecraft. But in his free-falling frame he falls through the event horizon unscathed. The dark experiment places an inherent limitation on the scope of what can be determined directly by experimentation. Clearly, the field should be aware of this limitation. Furthermore, a failure to admit to dark experiments has resulted in apparent paradoxes appearing in a diversity of contexts. In light of this, the purpose of the present paper is to formally and explicitly introduce the dark experi- ment.

Let $U=\left(m^{\prime}, m^{\prime \prime}\right)$ be an unordered pair of distinct massive objects in different reference frames. A dark experiment is an ordering $\left\langle m^{\mu}, m^{v}\right\rangle$ of the elements of $U$, either $\left\langle m^{\prime}, m^{\prime \prime}\right\rangle$ or, exclusively, $\left\langle m^{\prime \prime}, m^{\prime}\right\rangle$, where the left-hand member of the ordered pair is called the $o b$ server, and where

$$
\exists 1-\text { to }-1 f:\left\{\left\langle m^{\mu}, m^{v}\right\rangle\right\} \rightarrow\{\text { events }\}
$$

such that both elements of an ordered pair in a dark experiment agree on the events that unfold in the experiment.

Note that,

$$
\left\langle m^{\prime}, m^{\prime \prime}\right\rangle \neq\left\langle m^{\prime \prime}, m^{\prime}\right\rangle
$$

such that

$$
f\left(\left\langle m^{\prime}, m^{\prime \prime}\right\rangle\right) \neq f\left(\left\langle m^{\prime \prime}, m^{\prime}\right\rangle\right) .
$$

In other words, reality $f\left(\left\langle m^{\prime}, m^{\prime \prime}\right\rangle\right)$ for $m^{\prime}$ as the observer in the experiment $\left\langle m^{\prime}, m^{\prime \prime}\right\rangle$ is not the same as the reality $f\left(\left\langle m^{\prime \prime}, m^{\prime}\right\rangle\right)$ when $m^{\prime}$ is being observed in the experiment $\left\langle m^{\prime \prime}, m^{\prime}\right\rangle$. Consequently, the observer $m^{\prime}$ cannot determine directly from the experiment $\left\langle m^{\prime}, m^{\prime \prime}\right\rangle$ how the experiment $\left\langle m^{\prime \prime}, m^{\prime}\right\rangle$ unfolds, that is, 
without invoking some theory such as a conservation law or transformation algorithm. Of course, as the observer in the experiment $\left\langle m^{\prime \prime}, m^{\prime}\right\rangle, m^{\prime \prime}$ directly observes how that experiment unfolds. But he cannot communicate this to the observer $m^{\prime}$ because in the experiment $\left\langle m^{\prime}, m^{\prime \prime}\right\rangle$, $m^{\prime \prime}$ agrees with $m^{\prime}$ as to the events

$f\left(\left\langle m^{\prime}, m^{\prime \prime}\right\rangle\right) \neq f\left(\left\langle m^{\prime \prime}, m^{\prime}\right\rangle\right)$ that unfold in the experiment $\left\langle m^{\prime}, m^{\prime \prime}\right\rangle \neq\left\langle m^{\prime \prime}, m^{\prime}\right\rangle$.

Now while

$$
\begin{aligned}
& \left\{f\left(\left\langle m^{\prime}, m^{\prime \prime}\right\rangle\right) \cup f\left(\left\langle m^{\prime \prime}, m^{\prime}\right\rangle\right)\right\} \\
& -\left\{f\left(\left\langle m^{\prime}, m^{\prime \prime}\right\rangle\right) \cap f\left(\left\langle m^{\prime \prime}, m^{\prime}\right\rangle\right)\right\} \neq \varnothing
\end{aligned}
$$

is mathematically inherent in a dark experiment, no inconsistency arises if we choose to assume

$$
\left\{f\left(\left\langle m^{\prime}, m^{\prime \prime}\right\rangle\right) \cap f\left(\left\langle m^{\prime \prime}, m^{\prime}\right\rangle\right)\right\} \neq \varnothing .
$$

In the thought experiment involving a weatherman who jumps out of a spacecraft that is accelerating in the vicinity of a black hole, inequality (5) reflects the fact that the weatherman's radio signal is always lost when he falls through the event horizon, either because he and his equipment are vaporized by the stretched horizon or because the signal is trapped inside the black hole. In order to see what else this does and does not mean, let $m^{\prime}$ be a particle detector at rest on the surface of the Earth and let $m^{\prime \prime}$ be an exotic, short-lived particle in a cosmic ray shower. In the dark experiment $\left\langle m^{\prime}, m^{\prime \prime}\right\rangle$, the fact that the particle $m^{\prime \prime}$ is detected by $m^{\prime \prime}$ means that in the frame of the Earth the particle must have survived longer than its proper survival time would allow. This provides direct evidence for relativistic time dilation. But it tells us nothing directly about Lorentz contraction in the other frame, that is, in the experiment $\left\langle m^{\prime \prime}, m^{\prime}\right\rangle$. If we accept Lorentz contraction for purposes of inequality (5), then the difference between $f\left(\left\langle m^{\prime}, m^{\prime \prime}\right\rangle\right)$ and $f\left(\left\langle m^{\prime \prime}, m^{\prime}\right\rangle\right)$ is only that the particle ages faster in its own frame but spends proportionally less time traveling due to the shorter path. As a result, it is the same age when detected in each frame.

There is a conceptual reason to consider a more limited view that lends some credence to the famous twin paradox of special relativity. If space and time are truly unified in the 4-tuple we call spacetime, then speed $v$ through space should be monotone increasing as dimensionless, nonlinear speed through time,

$$
\gamma=\left[1-v^{2} c^{-2}\right]^{-\frac{1}{2}} \text {. }
$$

Under this interpretation of (6) it is not enough to say that moving clocks run slow but the effect cancels out because in the frame of the clock the duration of an experiment is shortened proportionally. It prevents asymmetric aging based on relativistic speed. Of course, this is precisely what modern cosmology assumes: that the cosmos and everything in it has aged for a fixed number of giga-years since the Big Bang. Putting aside speculations about General Relativity, this would have an unfortunate consequence for futurists. No matter how powerful our propulsion methods might be we could not travel into the future by cruising through space at a relativistic speed, something Carl Sagan [3] among others believed in. When we returned to our home world we would find that it had not aged any more than we had. This is because in our own reference frame the journey through space did not last all that long and was shortened just enough to cancel out the effect of our faster running clocks.

On the other hand if we accept Equation (6) as speed through time and allow asymmetric aging, then we must limit Lorentz contraction in the cosmic ray experiment. This is easily done by staying in the frame of the Earth so that Lorentz contraction simply alters the shape of the particle and not the duration of the experiment. For, suppose in the experiment $\left\langle m^{\prime}, m^{\prime \prime}\right\rangle$ there is a third identical but colder particle $m^{\dagger}$ generated in the laboratory. Then $\mathrm{m}^{\dagger}$ will decay at its proper rate while $\mathrm{m}^{\prime \prime}$ will be younger and last longer akin to the twin paradox. As of this writing there is a certain reason that it would be convenient to constrain Lorentz contraction. If Equation (6) is speed through time, then a particle moving at the speed of light would be moving infinitely fast through time. What better way to convince those who need to know that nothing could move faster? This would also allow us to define a rest mass as a particle that has a finite speed through time, perhaps because of the Higgs field if there is one. Since it would take an infinite amount of time to accelerate a rest mass to an infinite speed through time, dynamic mass indicates that inertia for space is also inertia for time. The annihilation of an electron and a positron could then be deemed to jump rest mass instantaneously to an infinite speed through time. Recent results from Ogonowski [4] suggest all this could also have implications for gravity.

\section{REFERENCES}

[1] A. D. Allen, "The Weatherman Who Fell down a Black Hole: What He Can Teach us about Reality,” Physics Essays, Vol. 25, No. 1, 2012, pp. 76-83. doi:10.4006/0836-1398-25.1.76

[2] L. Susskind, “The Black Hole War: My Battle With Stephen Hawking to Make The World Safe for Quantum Mechanics," Little Brown and Company, New York, 2008.

[3] C. Sagan, "Contact," Simon and Schuster, New York, 1985.

[4] P. Ogonowski, “Time Dilation as Field,” Journal of Modern Physics, Vol. 3, No. 2, 2012, pp. 200-207. doi:10.4236/jmp.2012.32027 